For personal use only. Not to be reproduced without permission of the publisher (editorial@gabi-journal.net).

\title{
US FDA proposals for naming of biologicals and labelling of biosimilars
}

\begin{abstract}
There is considerable concern and debate over the naming of biologicals and labelling of biosimilars. To ensure that patients receive the safest and most effective treatment, it is paramount that clinicians, prescribers and patients themselves have the relevant information regarding drug treatment. In response to concerns over how biologicals and biosimilars should be named and labelled, the US Food and Drug Administration has issued two sets of guidances that are set to pave the way for consistent and unified product naming and labelling.
\end{abstract}

\section{Keywords: Biologicals, biosimilars, labelling, naming, nomenclature, regulation}

\section{Pharmaceutical naming convention: the background}

The World Health Organization (WHO) established the use of international non-proprietary names, or INNs, that are assigned to active pharmaceutical substances, in the 1950s. These INNs are used in drug regulation, prescribing, labelling, dispensing, pharmacopoeias, pharmacovigilance and in scientific literature. For chemical pharmaceuticals, the assignment of INNs has been relatively straightforward to date [1].

In general, an INN will contain some information related to the active substance in the product and, as such, the word it forms will be uncommon or unfamiliar. It is typically made up of a prefix of up to three syllables, which is followed by a suffix. The suffix is also a stem that indicates any chemical and/or pharmacological relationships between products. Additionally, there may be sub-stems that give further drug-relationship information. When new drugs are developed that do not belong to a known stem, a new suffix is created [1].

Biological drugs, or biologicals, are complex biological molecules. As a result, these have required the creation of new stems and new naming methods or guidelines. The process of naming biosimilars has further increased naming complexity and to date, many different and inconsistent conventions have been applied worldwide [2]. Often, a biosimilar may share the same name as the reference product, and this, together with naming inconsistencies, has led to concern over the strength of the INN system currently in place [2].

\section{US FDA naming and labelling guidances}

The US Food and Drug Administration (FDA) has issued two draft guidance documents [3-5] in which it outlines how non-proprietary (un-trademarked) biological products should be named, and how biosimilar products should be labelled. At present, the guidances are not finalized and FDA is receiving input from a variety of stakeholders prior to finalization.

This paper summarizes the draft naming and labelling guidances and has been prepared based on the presentation of $\mathrm{Dr}$ Leah Christl from FDA [6].

\section{Biological nomenclature and FDA}

FDA aims to introduce the use of proper names for all biological products [3]. Through this, the use of designated suffixes for individual biological products, irrespective of their licensure pathway, is hoped to become routine in ordering, prescribing, dispensing, record-keeping and pharmacovigilance practices. This nomenclature is outlined in detail below. It is also thought that this will aid in prevention of the development of inaccurate perceptions related to the safety and efficacy of such products that can arise from knowledge of their licensure pathway.

\section{FDA position on ensuring product safety}

Product safety is of paramount importance in the treatment of patients. FDA has outlined that it is vital that originator biological products, related biological products and biosimilar products can be distinguished. This is because related biological products may be licensed for different indications, and biosimilar products are not always licensed for all originator indications. In addition, related biological products and biosimilar products may not be licensed for all the routes of administration and may be packaged in different delivery systems, e.g. pre-filled syringe instead of a vial, to that of the originator product is.

Through the inclusion of FDA designated suffixes, inadvertent substitution of products can be avoided. FDA states that the products will be distinguishable and so, only products that have been approved as interchangeable biologicals or biosimilars for a particular indication, will be used when switching treatments. This will also avoid any accidental alternating between different biological products that share the same name.

\section{FDA position on enhancing product pharmacovigilance}

Pharmacovigilance practices monitor the effects of products after licencing. This ensures that previously unreported adversities can be identified, monitored and evaluated. Traditionally, products are identified by proprietary name, non-proprietary name, manufacturer, national drug code (NDC) number, lot number, and billing codes. However, for both active and passive pharmacovigilance, it can be difficult to track adverse events and determine the product manufacturer, site of production, or lot, when a biological product has the same proper name as other biological products. Through the application of proper names, as described by the FDA guidance [3], the designated suffixes are hoped to facilitate the precise identification of a biological that has been associated with any adverse events. This will ensure improved knowledge and understanding of safety issues and allow for a more targeted agency response.

FDA proposal for standardized nomenclature of biologicals FDA defined how biologicals should be named in the draft guidance: Nonproprietary Naming of Biological Products [3]. 
This draft guidance states that each product will have a proper name that is made up of a core name, hyphenated to a suffix that is composed of four lowercase letters:

$$
\text { Proper name: core name + suffix }
$$

The core name is shared among biological products that contain related drug substances, e.g. filgrastim, epoetin alpha, in a similar manner to the stems of INNs. The four lowercase letters that make up the suffix should be unique to the product and not have any known meaning.

For example, for products that share the fictitious core name biologicamab, the proper names would be as follows:

- Originator biological product: biologicamab-cznm

- Related biological product: biologicamab-rzbh

- Biosimilar product: biologicamab-bixf

In addition to the requirements of the suffix as outlined above, FDA has laid out guidelines for what the suffix should not contain or resemble. It should not be in any way promotional, and as such have the potential to be misleading in terms of safety and efficacy. It is also important to ensure that suffixes are not to be confused with other elements of a prescription and therefore should not be the same, or too similar to, other abbreviations used in clinical and medical practice. They should not contain or imply any drug name or core name that has already been designated by the United States Adopted Name (USAN) council. To further reduce the risk of any potential medical or prescription errors, the name should not contain, or be similar to, the name of any other drug product currently on the market, nor should it have a suffix too similar to any other product's suffix designation.

FDA proposes that this convention should be applied to all biological products licensed under the Public Health Service (PHS) Act. As such, this would include both previously licensed and newly licensed products, together with innovator, related biological and biosimilar products. However, there is still some debate surrounding whether the non-proprietary name of an interchangeable product should have a unique suffix, or share that of its reference product.

Within the draft guidance, FDA put forward some product exceptions that would not need to use the naming convention. These are outlined by this statement: 'The draft guidance does not apply to biological products for which a proper name is provided in the regulations, e.g. 21 CFR part 640, or to certain categories of biological products for which there are well-established, robust identification and tracking systems to ensure safe dispensing practices and optimal pharmacovigilance (ISBT 128 for cord blood products)' [6].

\section{The need for regulated biologicals labelling}

There is also concern over the labelling of both biologicals and biosimilars. With respect to biosimilars, new and consistent methods of naming need to be devised in order to avoid incorrect drug administration, increase pharmacovigilance and also facilitate education of biosimilar usage. Product labelling exists to communicate information related to a product's safety and effectiveness to healthcare providers. It is thus vital that it is clear, understandable and specific, to ensure adequate treatment.

The educational requirement has been evidenced in a number of studies, including a recent survey in which physicians in Europe were questioned on their familiarity with biosimilar medicines [7]. The outcome of the study highlighted the need for distinguishable non-proprietary names to be given to all biologicals, including biosimilars. This is of particular importance as biosimilars differ from reference products in their structures but the active substance of a biosimilar has the same amino acid sequences [8]; and may not be approved for all the indications approved for the reference product. This is of particular importance for clinicians and patients who are considering switching drugs, and correct and adequate information on labels will facilitate a safe switch.

\section{Labelling biosimilars in Europe}

Biosimilar use was approved in Europe prior to approval in the US. However, no naming convention or labelling outline for biosimilars has been established in Europe to date. In 2015, a survey was conducted by the European Association for Bioindustries (EuropaBio) in which physicians expressed an explicit desire for more information to be displayed on the labels of biosimilar products [9]. In February 2016, a multi-stakeholder meeting was held by the European Biopharmaceutical Enterprises (EBE) and EuropaBio, in which aspects of the requirements for consistent and understandable labelling of biosimilars were discussed. Again, a major focus of this meeting was to ensure that clinicians, prescribers and/or patients receive adequate information to ensure safe and efficient use of the drug products [10].

\section{Labelling biosimilars in the US FDA biosimilar labelling guidance}

The general principles behind prescription drug labelling are to provide a summary of the essential scientific information needed by healthcare practitioners for the safe and effective use of a drug, and the labelling reflects FDA's finding of safety and effectiveness for the drug under the labelled conditions of use and facilitates prescribing decisions, thus enabling the safe and effective use of drugs and reducing the likelihood of medication errors.

Biosimilar products are developed to demonstrate biosimilarity to reference licensed products. As such, data supporting the licensure of biosimilars would generally not be designed to independently demonstrate safety and efficacy. In March 2016, FDA issued a draft guidance on Labeling for Biosimilar Products [4]. FDA has outlined recommendations for the product labelling of biosimilars pertaining only to the prescribing information to ensure patients are well-informed with regard to the products available to them. In the draft guidance, it is proposed that those submitting biosimilar product applications under section $351(\mathrm{k})$ should develop draft labels which incorporate the data and information from the reference product labelling, with the addition of any biosimilar product-specific modifications, specific labelling recommendations for interchangeable biological products are not provided in this guidance.

\section{Biosimilarity statement}

FDA has recommended the inclusion of a biosimilarity statement. This should be on the line immediately beneath the 
initial US approval date in Highlights, that informs the product is biosimilar to the reference product. A sample of such a statement is given below:

'BIOSIMILAR PRODUCT'S PROPRIETARY NAME (biosimilar product's proper name)] is biosimilar* to [REFERENCE PRODUCT'S PROPRIETARY NAME (reference product's proper name)] for the indications listed.' (1).

The asterisk is to draw attention to the footnote that states: *Biosimilar means that the biological product is approved based on data demonstrating that it is highly similar to an FDAapproved biological product, known as a reference product, and that there are no clinically meaningful differences between the biosimilar product and the reference product.

The guidance also indicates that the statement should conclude with a cross-reference to the INDICATIONS AND USAGE section '(1)' the Full Prescribing Information, which contains further, more detailed information.

Example of the statement of a fictitious product NEXSYMEO:

NEXSYMEO (replicamab-cznm) is biosimilar* to JUNEXANT (replicamab-hjxf) for the indications listed. (1)

\section{Specific recommendations for labelling of biosimilar}

The relevant data and information from the reference product labelling that should be incorporated into the biosimilar product labelling will depend on whether the applicant is seeking approval for all conditions of use, e.g. indication(s), dosing regimen(s), or fewer than all conditions of use of the reference product for the biosimilar product. As such, FDA makes further recommendations to take this into account in the biosimilar labelling.

For biosimilar containing certain differences to the reference product, the labelling may include information specific to the biosimilar product in which differences in administration, preparation, storage, or safety information that do not otherwise preclude a demonstration of biosimilarity. A biosimilar product label may also include its conformity to the Physician Labelling Rule (PLR) and/or the Pregnancy and Lactation Labelling Rule (PLLR), as reference product labelling may not be required to conform to these requirements.

Where the biosimilar product labelling is based on the reference product labelling, text can be similar but need not be identical and should reflect the currently available information necessary for the safe and effective use of the biosimilar product.

\section{Use of biosimilar/reference product name and core name in labelling}

As is outlined for all biological products identification, the proper names will be composed of the core name and a unique suffix. Identification of a specific product, whether that be by biosimilar product name, reference product name or core name, will be dependent on the context in which the information is presented.

\section{Use of biosimilar/reference product name}

If the biosimilar product has a proprietary name, FDA recommends that the proprietary name be used.
If a proprietary name is not available for the biosimilar product, the biosimilar product's proper name (the non-proprietary name designated by FDA in the licence for a biological product licensed under the PHS Act) should be used.

FDA recommends the use of the biosimilar product name in circumstances that:

- information described is specific to the biosimilar product, for instance, the sections of INDICATIONS AND USAGE, DOSAGE AND ADMINISTRATION, DOSAGE FORMS AND STRENGTHS, DESCRIPTION, and HOW SUPPLIED/STORAGE AND HANDLING.

- directive statements and recommendations for preventing, monitoring, managing, or mitigating risks, for instance, statements typically included in the BOXED WARNING, CONTRAINDICATIONS, WARNINGS AND PRECAUTIONS, and DRUG INTERACTIONS sections.

FDA states that the reference product's proper name should be used when clinical studies or data derived from studies with the reference product, e.g. adverse reactions, clinical studies, are described in the biosimilar product labelling.

\section{Use of core name}

FDA also outlined that the core name should be used in labelling when overall risks and benefits profile of the reference product is also relevant to the biosimilar product. This should include the risk of any adverse reactions or effects seen following administration of the reference product, even if these are not known to be observed with the biosimilar product.

In the labelling section, when a risk applies to both the biosimilar product and reference product, FDA advises that the core name of the reference product be used with the addition of the word 'products', i.e. replicamab products, to imply that the information is relevant to both products. This would be included in sections such as a boxed warning, contraindications, warnings and precautions, adverse reactions (post-marketing experience).

\section{Use of more than one product name}

FDA notes that there are circumstances in which it may be necessary to use more than one form of the product identification approaches in the labelling to accurately convey information. An hypothetical example where the use of the three naming approaches is presented below:

\section{'Replicamab products can cause hepatoxicity and acute bepatic failure. In clinical trials of replicamab-hjxf, 10\% of patients developed elevated ALT or AST greater than three times the upper limit of normal and 5\% progressed to acute bepatic failure. Evaluate serum transaminases (ALT and AST) and bilirubin at baseline and montbly during treatment with NEXSYMEO ...'}

When considering which name to use - biosimilar product name, reference product name or core name - FDA advises:

'All text in biosimilar product labeling, even sections that have been based on reference product labeling, should be carefully evaluated for the most appropriate product identification approach.' 


\section{FDA's opinion of their labelling approach}

With respect to FDA's approach on biosimilar labelling, Dr Leah Christl, Associate Director for Therapeutic Biologics and lead of the Therapeutic Biologics and Biosimilars Staff in the Office of New Drugs, stated 'We think that our approach to biosimilar labeling will be truly beneficial to healthcare providers as they consider prescribing options and the risk-benefit decisions for their patients. The biosimilar labelling guidance has been issued in draft to provide an opportunity for public comment and we hope to hear from the various stakeholder communities industry, healthcare providers and patients. We will review and consider all of the comments received as we work to finalize guidance on this topic' [11].

\section{Summary}

In recent years, the naming of biological and biosimilar products has become increasingly complex and is inconsistent throughout the world. This has also led to confusion over how such products should be labelled, and there is particular concern over differentiation between biosimilar products and their reference products in labelling.

FDA has issued two draft guidance documents in which they outline the proposed methods for biological product naming and biosimilar product labelling. The guidances are comprehensive and convey clear instructions for naming and labelling, to facilitate the use of these products and ensure correct administration to patients. They aim to be able to give all required information to clinicians and patients to ensure the safe and effective use of products.

At present, the guidances are in draft format and FDA hopes to receive feedback on them from a variety of stakeholders before the nomenclature and labelling guidelines are finalized.

\section{Competing interests: None.}

Provenance and peer review: Article prepared based on the presentation of Dr Leah Christl, US FDA; internally peer reviewed.

Alice Rolandini Jensen, MSci, GaBI Journal Editor

\section{References}

1. Robertson JS. The challenges of nomenclature - INN, biosimilars and biological qualifiers. Generics and Biosimilars Initiative Journal (GaBI Journal). 2015;4(3):110-2. doi:10.5639/gabij.2015.0403.025

2. Alexander EA. The biosimilar name debate: what's at stake for public health. Generics and Biosimilars Initiative Journal (GaBI Journal). 2014;3(1):10-2. doi:10.5639/gabij.2014.0301.005

3. U.S. Food and Drug Administration. Guidance for industry. Nonproprietary naming of biological products. August 2015 [homepage on the Internet]. [cited 2016 Sep 15]. Available from: http://www.fda.gov/downloads/Drugs/ GuidanceComplianceRegulatoryInformation/Guidances/UCM459987.pdf

4. U.S. Food and Drug Administration. Guidance for industry. Labeling for biosimilar products. March 2016 [homepage on the Internet]. [cited 2016 Sep 15]. Available from: http://www.fda.gov/downloads/drugs/guidancecomplianceregulatoryinformation/guidances/ucm493439.pdf

5. GaBI Online - Generics and Biosimilars Initiative. FDA issues draft guidance on naming biologicals [www.gabionline.net]. Mol, Belgium: Pro Pharma Communications International; [cited 2016 Sep 15]. Available from: www. gabionline.net/Guidelines/FDA-issues-draft-guidance-on-naming-biologicals

6. Christi L. U.S. Food and Drug Administration update: implementation of the Biologics Price Competition and Innovation Act of 2009. 14th Annual Biosimilar Medicines Group Conference; 28-29 April 2016; London.

7. Dolinar RO, Reilly MS. Biosimilars naming, label transparency and authority of choice - survey findings among European physicians. Generics and Biosimilars Initiative Journal (GaBI Journal). 2014;3(2):58-62. doi:10.5639/gabij.2014.0302.018

8. Kurki P. Biosimilars for prescribers. Generics and Biosimilars Initiative Journal (GaBI Journal). 2015;4(1):33-5. doi:10.5639/gabij.2015.0401.008

9. GaBI Online-Generics and Biosimilars Initiative. Doctors want more details in biosimilars labelling [www.gabionline.net]. Mol, Belgium: Pro Pharma Communications International; [cited 2016 Sep 15]. Available from: www.gabionline. net/Biosimilars/Research/Doctors-want-more-details-in-biosimilars-labelling

10. EuropaBio 2015 [homepage on the Internet]. [cited 2016 Sep 15]. Available from: http://www.europabio.org/healthcare-biotech/publications/results-firstever-european-biosimilars-labelling-survey

11. U.S. Food and Drug Administration. Christi L. From our perspective: biosimilar product labelling [homepage on the Internet]. [cited 2016 Sep 15]. Available from: http://www.fda.gov/Drugs/NewsEvents/ucm493240.htm

DOI: $10.5639 /$ gabij.2016.0503.036

Copyright $\odot 2016$ Pro Pharma Communications International 